und Rechtsanwälte wurden von den Nationalsozialisten als „nichtarisch“ definiert. Ein Drittel der deutschen Juden wohnte in Berlin. Daher gab es dort auch die meisten jüdischen Rechtsanwälte und Rechtsanwältinnen. Es waren am 1. Januar 1933 etwa 1.800 Anwältinnen und Anwälte von insgesamt 3.400 Berliner Anwältinnen und Anwälten.

Etwa 400.000 Deutschen jüdischer Herkunft gelang es, das Deutsche Reich rechtzeitig zu verlassen und am Leben zu bleiben. Ungefähr 15.000 deutsche Juden überlebten die Konzentrationslager, im Untergrund oder als Ehepartner/in von Nichtjuden. Nach 1945 kehrte nur eine vergleichsweise geringe Zahl aus der Emigration nach Deutschland zurück. Die meisten zogen es vor, Deutschland fern zu bleiben. Bis 1989 lebten nicht mehr als 30.000 Juden in Deutschland. Da Juristinnen und Juristen jedoch größere Schwierigkeiten haben, ihren Beruf in einem anderen Land mit fremder Sprache auszuüben als etwa Ingenieur/inn/e/n oder Ärzt/inn/e/n, dürfte auch der Rückkehreranteil unter den Juristen entsprechend höher sein. Hierzu ein
Zitat von Erna Proskauer, das auch Hans Bergemann wiedergibt: „Gerade Juristen mit ihrem örtlich begrenzten beruflichen Anwendungsgebiet fanden nirgends im Exil, vor allem aber nicht in den engen Grenzen der israelischen Wirtschaft, eine Erwerbsmöglichkeit, geschweige denn eine angemessene.“ 349 ist nicht viel. Zumal sich von 1945 bis 1990 nur 179 Anwält/inn/ e/n jüdischer Herkunft zeitweise oder dauerhaft (wieder) in Berlin niederließen, die 1933 bereits als Anwält/inn/e/n oder Beamt/ inn/e/n im höheren Justizdienst tätig gewesen waren oder sich 1933 in ihrer juristischen Ausbildung befunden hatten. Und auch das Kapitel, das über die Grundlagen der Rückerstattung und Entschädigung für die verfolgten jüdischen Rechtsanwältinnen und Rechtsanwälte berichtet, ist deprimierend.

Die Rechtsanwaltskammer Berlin hofft, mit diesem Band dazu beizutragen, dass das Schicksal der jüdischen Kolleginnen und Kollegen nicht vergessen wird und auch künftigen Generationen zugänglich bleibt. Dieser Hoffung schließe ich mich an. Anke Gimbal, Berlin

\title{
Rezension:
}

\section{Rechtsnormen des StGB suchen, verstehen und auslegen}

\section{Otto Lagodny}

Rechtsnormen des StGB suchen, verstehen und auslegen

Eine Anleitung für die ersten Schritte im Strafrecht

2012, Buch, $203 \mathrm{~S}$.

nwVerlag IDBN 978-3-7083-0860-9

Wien, Graz 2012

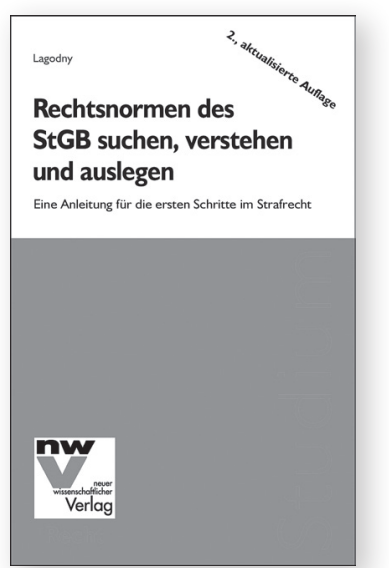

Bei dem Buch des Salzburger Professors Otto Lagodny handelt es sich um ein Lehrbuch für Studierende der Rechtswissenschaft in den ersten Semestern. Es muss allerdings zu Beginn angemerkt werden, dass das Buch für österreichische Studierende verfasst wurde und vor allem für diese nützlich ist, da es sich auf österreichische Rechtsnormen bezieht

Das Buch ist in mehrere Abschnitte unterteilt: Nach einer Einführung gibt es ein Kapitel über das Finden einer Norm im StGB, eines über das Lesen und Verstehen der gefundenen Norm, ein weiteres über das strafrechtsbezogene Lesen und ein abschließendes kurzes Kapitel, das sich mit dem schnellen Wieder-Erkennen der Normen befasst.

In dem Kapitel über das Finden einer Norm stellt der Autor zunächst den Aufbau des Strafgesetzbuches dar und erläutert kurz den Inhalt der einzelnen Abschnitte. Er gibt zahlreiche „Suchbeispiele“, wie er es nennt, dafür, wie man eine be- stimmte Norm auffinden kann, all dies anhand kleiner Fälle, deren Lösung am Ende präsentiert wird.

Der Abschnitt über das Lesen und Verstehen der gefundenen Norm befasst sich in erster Linie mit den Auslegungsmethoden von Rechtsnormen. Diese werden anhand einer größeren Auswahl von Strafrechtsnormen des StGB erläutert und mit Beispielen unterlegt.

Das Kapitel über strafrechtsbezogenes Lesen stellt die Verbindung zwischen Allgemeinem und Besonderem Teil des StGB her, wobei der Allgemeine Teil als „Werkzeugkasten“ für den Besonderen Teil bezeichnet wird. Auch hier wird umfangreich auf Normen und Beispiele Bezug genommen. Dabei wird in gebotener Kürze der Allgemeine Teil abgehandelt, so dass den Studierenden ein erster Eindruck über diesen vermittelt wird

Im letzten Abschnitt, der das Wieder-Erkennen von Normen zum Inhalt hat, geht es in erster Linie um die Anwendung des in den vorherigen Kapiteln gelernten im Rahmen einer Klausur. Hier soll vermittelt werden, wie die Studierenden auch unter Zeitdruck die Normen schnell und effizient wiederfinden und anwenden können. Auch die Vorbereitung der Klausur durch Unterstreichung der wesentlichen Bestandteile einer Rechtsnorm wird dabei abgehandelt.

Insgesamt ist das Buch für Studienanfänger/innen sicherlich empfehlenswert, weil eine sichere Beherrschung der Methodik eine unerlässliche Grundlage für ein erfolgreiches Studium der Rechtswissenschaften ist und diese hier umfassend dargestellt wird. Die Besonderheit dabei ist, dass die Methodik hier ganz speziell auf das Strafrecht abgestimmt wurde. Ein wenig negativ fällt dabei allerdings auf, dass in dem Werk bereits im- 
mens viele Normen des Besonderen Teils des Strafgesetzbuches abgehandelt werden. Dies könnte zu einer Überforderung der Studierenden führen, die sich in einem so frühen Stadium ihrer Ausbildung bereits mit derart vielen Normen befassen sollen.

Für österreichische Studierende ist das Buch sicherlich empfehlenswert. Für diejenigen in Deutschland ist es eher nicht nutzbar, denn obwohl die Methodik selbstverständlich auch für sie außerordentlich wichtig ist, so sollten sie diese doch anhand der deutschen Rechtsnormen erlernen.

Katrin Stegemann-Siebert

Staatsanwältin, Kiel

\section{Erfolgreicher Start des Netzwerks für juristische Gleichstellungsbeauftragte}

\section{Eva Schübel \\ Vizepräsidentin des djb; Bundesanwältin beim BGH, Karlsruhe}

Die Gleichstellungsbeauftragten werden nicht selten mit der Bemerkung konfrontiert „Ist das überhaupt gleichstellungsrelevant? “, was heißen soll: Hat die Gleichstellungsbeauftragte da eigentlich mitzureden? Möglichst nicht und wenn, dann allenfalls durch Kenntnisnahme einer verfügten Maßnahme - dies ist eine nach wie vor bei Verwaltungen und Dienststellenleitungen weit verbreitete Auffassung. Die Gleichstellungsgesetze werden nicht selten als quantité négligable betrachtet und beiseite gelassen. Dies ist allerdings nicht mehr gänzlich ungefährlich. Nach einer Entscheidung des Verwaltungsgerichts Frankfurt ${ }^{1}$ stellt die fehlende Mitwirkung der Gleichstellungsbeauftragten bei den in $\$ 19$ Absatz 1 BGleiG genannten personellen, organisatorischen und sozialen Angelegenheiten einen absoluten Verfahrensfehler dar.

Eine effektive Mitwirkung beansprucht freilich - wie bei den Personaldezernenten auch - Arbeitszeit. Sieht das Gleichstellungsgesetz wie in einzelnen Bundesländern keine Entlastung der Gleichstellungsbeauftragten von den anderweitigen Dienstgeschäften vor oder nimmt diese die gesetzlich vorgesehene Möglichkeit lieber nicht in Anspruch, wird sich nicht lange nach der Wahl Frust einstellen: Wem praktisch keine Zeit für die Amtsausübung bleibt, der kann keinen Einfluss geltend machen. Eine oft geäußerte Klage bei den Brühler Gleichstellungstagen, die ich aus eigener Erfahrung bestätigen kann. Wer das Amt der Gleichstellungsbeauftragten neben einer Vollzeittätigkeit mit erledigt, kann nur bei sehr wenigen Personalentscheidungen nachhaken, weil schon der Aktenumlauf und die unbedingt erforderlichen Gespräche und Besprechungen viel Zeit beanspruchen. Nur mit einem nicht zu knapp bemessenen Freiraum kann die Gleichstellungsbeauftragte ihre Mitwirkungsrechte effektiv durchsetzen und die gesetzlich vorgesehenen Controllingaufgaben wahrnehmen - denn ablehnende Stellungnahmen zu fertigen und Einsprüche zu begründen, kostet Zeit. Dann allerdings bläst ihr meist kräftiger Wind entgegen und sie benötigt neben einem breiten Kreuz auch juristischen und moralischen Beistand von Kolleginnen. Aus dieser Erkenntnis ist bei mir die Idee entstanden, Gleichstellungsbeauftragte zu vernetzen, die wie ich Juristinnen sind.
Für die Gründung eines solchen Netzwerks ist die Zeit offensichtlich reif gewesen. Seit der Billigung des Projekts durch den djb-Bundesvorstand im November 2011 haben wir dafür schon 48 Juristinnen gewinnen können, die als Gleichstellungs- bzw. Frauenbeauftragte tätig sind oder sich hauptberuflich mit Gleichstellungsaufgaben befassen. Am 24. August 2012 fand in Berlin das Gründungstreffen mit 17 Teilnehmerinnen statt, bei dem insbesondere die Arbeitsweise und Tätigkeitsfelder des Netzwerks vereinbart worden sind. Die Mitgliedsfrauen arbeiten per djb-Internetforum zusammen und treffen sich einmal im Jahr in Berlin. Wer in das Netzwerk aufgenommen werden möchte, muss Mitglied des djb sein, weil der djb für die Kosten aufkommt, insbesondere seine Geschäftsstelle die Organisation unterstützt. Das Netzwerk dient vorrangig dem fachlichen Austausch und der kollegialen Beratung zwischen seinen Mitgliedsfrauen. Wir wollen aber auch die Stellung, Aufgaben und Rechte der Gleichstellungsbeauftragten näher beleuchten, uns zu Gleichstellungsthemen aus juristischer Sicht äußern und Stellung zu Gesetzentwürfen im Gleichstellungsrecht in Abstimmung mit der jeweils zuständigen djb-Kommission nehmen. Durch Veröffentlichungen und die Zusammenarbeit mit Netzwerken, die ähnliche Ziele verfolgen, möchten wir die Ergebnisse unserer Arbeit anderen Gleichstellungsbeauftragten bekannt machen. Daneben werden wir den Kontakt in die Politik hinein suchen. Eine Sammlung von Urteilen, die auf Gleichstellungsrecht und das Amt der Gleichstellungsbeauftragten Bezug nehmen, und eine Liste von im Gleichstellungsrecht spezialisierten Rechtsanwältinnen und Rechtsanwälten sollen die Netzwerkarbeit unterstützen.

Das erste regelmäßige Treffen des Netzwerks fand am 9. November 2012 mit 17 Kolleginnen statt. Richter am Bundesverwaltungsgericht Prof. Dr. Jan Hecker referierte über die Entscheidung des Bundesverwaltungsgerichts vom 8. April $2010^{2}$ zum Teilnahmeanspruch der Gleichstellungsbeauftragten an Führungsklausuren ihrer Dienststelle. Für die Praxis lassen sich daraus kurz zusammengefasst folgende Schlüsse ziehen: $\mathbb{2} 20$ Absatz 1 Satz 3 BGleiG, wonach der Gleichstellungsbeauftragten Gelegenheit zur aktiven Teilnahme an allen Entscheidungsprozessen zu personellen, organisatorischen

1 Beschluss v. 4.10.2011, 9 L 2202/11.F.

26 C 3.09. 